in terms of the identification of accurate biomarkers for a specific cancer type. As we move into an era of personalized and precision medicine that involves deep and multi-omic profiling of each cancer patient, this current limitation can be addressed through large data sets and more advanced machine- and deep-learning algorithms, presuming there is a will among all concerned to share the required information more openly than is currently practised $^{7}$. As the current study has successfully demonstrated, the combination of a range of platforms is inevitably essential for more precise and informed diagnosis of cancers. A recent study utilized a combination of conventional tumour DNA assays, bioinformatics tools, as well as cognitive computational systems for the analysis of deep cancer genomic datasets to identify actionable variants and therapeutic options in a clinically timely manner for individual patients ${ }^{8}$.

Despite the limitations of these studies, such as the need for longer-term patient surveillance, they take a major step forward in highlighting the progress of cancer diagnosis, predicting prognosis and identifying patients that would likely require aggressive therapeutic options. With our aging populations comes the dramatic increase in the incidence of cancer. According to Cancer Research UK, one in two individuals will develop cancer at some point in their lives 9 . However, through the use of biomarkers such as ctDNA methylation markers and prediction of prognosis, the goal of defeating cancer, or at least turning it into a treatable disease through early detection, is evidently coming within reach.

Miljana Tanić and Stephan Beck are at the UCL Cancer Institute, University College London, London, WC1E 6BT, UK.

e-mail:s.beck@ucl.ac.uk

References

1. Xu, R.-h. et al. Nat. Mater. 16, 1155-1161 (2017).

2. Wan, J. C. M. et al. Nat. Rev. Cancer 17, 223-238 (2017).

3. Crowley, E., Di Nicolantonio, F., Loupakis, F. \& Bardelli, A. Nat. Rev. Clin. Oncol. 10, 472-884 (2013).

4. Bettegowda, C. et al. Sci. Transl. Med. 6, 224ra24 (2014).

5. Tanić, M. \& Beck, S. Curr. Opin. Genet. Dev. 42, 48-55 (2017).

6. Flavahan, W. A., Gaskell, E. \& Bernstein, B. E. Science 357, eaal2380 (2017)

7. Clinical Cancer Genome Task Team of the Global Alliance for Genomics and Health N. Engl. J. Med. 376, 2006-2009 (2017).

8. Wrzeszczynski, K. O. et al. Neurol. Genet. 3, e164 (2017).

9. Ahmad, A. S., Ormiston-Smith, N. \& Sasieni, P. D. Br. J. Cancer. 112, 943-947 (2015)

\section{GET A GRIP}

Given how much we navigate the world by touch, it's surprising how reliant on empiricism an understanding of haptic sensation is. It's a complex interface that our skin makes with the world, in comparison with our optic, auditory, olfactory and gustatory sensory apparatus. With all of those senses, the brain does a great deal with the raw neural signal, not least in simplifying by categorization: making distinctions of colour or basic tastes such as sweet or sour, say. Some haptic categories are clear enough: rough/smooth, hot/cold, hard/soft. But we make subtler distinctions between the feel of materials too, the criteria for which are less obvious.

Such evaluations are crucial in efforts to create haptic touchscreens, for which mechanical manipulations such as vibrations might be used to elicit a different tactile sensation from the same material substrate. How can illusions of this kind be generated?

That task looks somewhat harder in the light of the finding of Dzidek et al. that touch contact may have substantial time dependence ${ }^{1}$. We might be inclined naively to imagine that a finger pressed to a glass screen establishes a contact that remains subsequently unchanging. But Dzidek et al. show that the contact area may evolve over tens of seconds - even though the apparent macroscopic area over which fingertip and glass maintain intimate contact stays the same.

The methodology is charmingly simple in principle: the researchers measure the time evolution of fingerprints on a glass prism, using total internal reflection. The prism is pressed onto the subjects' finger pads, rather than vice versa, to control the loading. While the gross contact area remains constant as soon as loading is completed, the actual contact area measured from image analysis of fingerprints slowly increases for up to 20 seconds afterwards, according to an exponential (first-order) rate law. The coefficient of friction increases in a concomitant manner, while the normal load declines.

The reason for these observations, Dzidek et al. conclude, is that the material properties of the skin itself the so-called stratum corneum, made up of the elastic protein keratin change over time. Keratin contains some proteins in a crystalline state interspersed among others that are amorphous, and the amorphous component is known to be sensitive to moisture ${ }^{2}$. As sweat is excreted between the skin and the glass surface, the keratin is therefore softened, bringing more of the ridges on the finger pad into direct contact.

This situation contrasts that for a soft elastomeric surface. Using the same techniques for a transparent

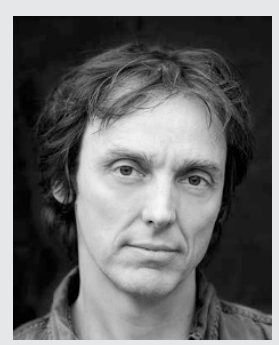

PHILIP BALL

silicone, the researchers show that the real contact area reaches its saturated value as soon as loading is complete and stays constant thereafter, as does the coefficient of friction. For this reason, we feel instantly that we have a good grip on a pen with a thin rubber sheath but not with one made from hard plastic or metal. The asymptotic coefficient of friction is also likely to be lower in the latter case.

One question left unanswered at this stage is whether these aspects of tactility are adaptive or incidental. Might there be some advantage to a touch interaction that slowly increases its friction for some surfaces? Or have we, conversely, evolved the materiality of our devices to suit our biology?

References

1. Dzidek, B., Bochereau, S., Johnson, S. A., Hayward, V. \& Adams, M. J. Proc. Natl Acad. Sci. USA 114, 10864-10869 (2017).

2. McKittrick, J. et al. JOM 64, 449-468 (2012). 